\title{
Lie Point Symmetries for Reduced Ermakov Systems
}

\author{
F. Haas* and J. Goedert \\ Centro de Ciências Exatas e Tecnológicas - UNISINOS \\ Av. Unisinos, 950 \\ 93022-000 São Leopoldo, RS, Brazil
}

November 12, 2018

\begin{abstract}
Reduced Ermakov systems are defined as Ermakov systems restricted to the level surfaces of the Ermakov invariant. The condition for Lie point symmetries for reduced Ermakov systems is solved yielding four infinite families of systems. It is shown that $S L(2, R)$ always is a group of point symmetries for the reduced Ermakov systems. The theory is applied to a model example and to the equations of motion of an ion under a generalized Paul trap.
\end{abstract}

PACS numbers: 02.30.Hg; 02.90.+p; 03.20.+i

Keywords: Ermakov system; Lie symmetry; Ermakov invariant.

\section{Introduction}

Ermakov systems [1]-3 play an important role in a variety of physical and mathematical situations. The most recent analyses involving Ermakov systems deal with Bose-Einstein condensates and cosmological models [4]- $[$ ], nonlinear supersymmetric Darboux transformations [7], the free fall of a

*ferhaas@exatas.unisinos.br 
quantum bouncing ball [8], conformal quantum mechanics 9] and generalized Hamiltonian structures [10]. From the theoretical viewpoint, Ermakov systems always admit a constant of motion, the Ermakov invariant, and are amenable to a nonlinear superposition law [11. In addition, Ermakov systems are linearizable under broad circumstances [12, 13.

As is well known, the group theoretic analysis of a dynamical system is a subject of great relevance [14] not only for the reduction of order and the search for invariants for the system but also for a better understanding of its structural properties. The point symmetry group of Ermakov systems has been identified as the $S L(2, R)$ group [15]-19]. More recently, using the converse to Noether's theorem, it has been shown that the Ermakov invariant can be associated to a dynamical symmetry, in the cases where the Ermakov system admits a variational formulation [20. In addition, $S L(2, R)$ has also been found [21] as the symmetry group of Kepler-Ermakov systems [22, which can be viewed either as perturbations of the planar Kepler problem or of the classical Ermakov system. The purpose of this paper is to follow this trend from a different perspective and study the Lie point symmetries of Ermakov systems restricted to manifolds where the Ermakov invariant has a fixed constant value. The importance of this study may not be underestimated since the existence of the Ermakov invariant is automatic.

In polar coordinates, the Ermakov system reads

$$
\begin{aligned}
\ddot{r}-r \dot{\theta}^{2} & +\omega^{2} r=\frac{F(\theta)}{r^{3}}, \\
r \ddot{\theta}+2 \dot{r} \dot{\theta} & =\frac{G(\theta)}{r^{3}}
\end{aligned}
$$

where $F$ and $G$ are arbitrary functions of the angle and $\omega$, in principle, can depend arbitrarily on the dynamical variables. More often, $\omega$ is a function of time only, in which case it has the interpretation of a time-dependent frequency. For simplicity, we consider the case $\partial \omega / \partial \dot{r}=0$. No mater the special form of $\omega$, the Ermakov systems always possess the constant of motion

$$
I=\frac{1}{2}\left(r^{2} \dot{\theta}\right)^{2}-\int^{\theta} G(\phi) d \phi
$$

the so-called Ermakov invariant [1]-3].

The existence of a constant of motion allows the reduction of the order of the system. More exactly, the fourth-order system (1-2) can be rewritten 


$$
\begin{aligned}
\ddot{r} & =A(r, \theta, t), \\
r^{2} \dot{\theta} & =B(\theta),
\end{aligned}
$$

where the functions $A$ and $B$ are defined according to

$$
\begin{aligned}
A(r, \theta, t) & =-\omega^{2} r+\frac{1}{r^{3}}\left(F(\theta)+2 I+2 \int^{\theta} G(\phi) d \phi\right), \\
B(\theta) & =\sqrt{2}\left(I+\int^{\theta} G(\phi) d \phi\right)^{1 / 2} .
\end{aligned}
$$

The fact that $\partial \omega / \partial \dot{r}=0$ ensures the indicated functional dependence of $A$ in (6). Notice, however, that $\omega$ can freely depend, for instance, on $\dot{\theta}$, since this dependence can be eliminated through (5). The choice $\partial \omega / \partial \dot{r}=0$ has a decisive influence on the simplification of the symmetry analysis so that we do not claim our results are the most general.

Equations (44) are a third-order dynamical system which we call the reduced Ermakov system. Even if we do not show explicitly, notice that the reduced equations do depend parametrically on $I$. Also, the function $B$ is not identically zero except in the trivial case $I=G(\theta)=0$, which we do not consider here.

The purpose of this work is to perform the Lie point symmetry analysis of reduced Ermakov systems. Since for Lagrangian Ermakov systems the Ermakov invariant is directly related to a dynamical Noether symmetry [20, it is to be expected that the algebra $\operatorname{sl}(2, R)$ do play a fundamental role on the reduced Ermakov system.

The organization of the paper is as follows. In Section 2, the general symmetry conditions to be satisfied by reduced Ermakov systems and his generator of symmetries are determined. In Section 3, we solve the symmetry conditions in the case of transformations of the time parameter not involving the dynamical coordinates. This yields four classes of reduced Ermakov systems admitting Lie point symmetries. In Section 4, we show that, irrespective of its specific form, the reduced Ermakov systems always do admit $S L(2, R)$ as a symmetry group. This shows that, more properly, $S L(2, R)$ is the group of symmetries for reduced Ermakov systems, the reduction being possible thanks to a dynamical Noether symmetry in the case of Lagrangian Ermakov systems. In Section 5, we apply the whole formalism to a particular example, looking for reduced Ermakov systems admitting 
a chosen Lie symmetry. In Section 6, we start from the equations for an ion under a generalized Paul trap and find the circumstances under which these equations, written as a reduced Ermakov system, do possess Lie point symmetries. Section 7 is devoted to the conclusions.

\section{Lie Symmetries}

Consider infinitesimal point transformations of the form

$$
\begin{aligned}
& \bar{r}=r+\varepsilon R(r, \theta, t), \\
& \bar{\theta}=\theta+\varepsilon S(r, \theta, t), \\
& \bar{t}=t+\varepsilon T(r, \theta, t),
\end{aligned}
$$

for functions $R, S$ and $T$ to be determined and infinitesimal parameter $\varepsilon$. The procedure for computing Lie symmetries is well known [14] and we limit ourselves to sketch the critical steps in our case. The above infinitesimal transformation will be a Lie point symmetry of the reduced Ermakov system if and only if (4/5) remains formally invariant under (8),10) up to first order in $\varepsilon$, in the solution set of the reduced Ermakov system. This symmetry condition will imply the vanishing of two separate polynomials in $\dot{r}$, one associated with the radial equation (44), the other associated with the angular equation (5). Assuming the vanishing of the coefficient of different powers of $\dot{r}$ we get the following set of linear, coupled partial differential equations,

$$
\begin{aligned}
\frac{\partial^{2} T}{\partial r^{2}} & =0 \\
\frac{\partial^{2} R}{\partial r^{2}} & -\frac{2 B}{r^{2}} \frac{\partial^{2} T}{\partial r \partial \theta}-2 \frac{\partial^{2} T}{\partial r \partial t}+\frac{2 B}{r^{3}} \frac{\partial T}{\partial \theta}=0 \\
\frac{2 B}{r^{2}} \frac{\partial^{2} R}{\partial r \partial \theta} & +2 \frac{\partial^{2} R}{\partial r \partial t}-\frac{2 B}{r^{3}} \frac{\partial R}{\partial \theta}-3 A \frac{\partial T}{\partial r}-\frac{B B^{\prime}}{r^{4}} \frac{\partial T}{\partial \theta} \\
& -\frac{B^{2}}{r^{4}} \frac{\partial^{2} T}{\partial \theta^{2}}-\frac{2 B}{r^{2}} \frac{\partial^{2} T}{\partial \theta \partial t}-\frac{\partial^{2} T}{\partial t^{2}}=0 \\
r^{2} \frac{\partial S}{\partial r} & -B \frac{\partial T}{\partial r}=0 \\
B \frac{\partial S}{\partial \theta} & -S B^{\prime}+r^{2} \frac{\partial S}{\partial t}-\frac{B^{2}}{r^{2}} \frac{\partial T}{\partial \theta}-B \frac{\partial T}{\partial t}+\frac{2 R B}{r}=0 \\
R \frac{\partial A}{\partial r} & +S \frac{\partial A}{\partial \theta}+T \frac{\partial A}{\partial t}=\left(\frac{\partial R}{\partial r}-\frac{2 B}{r^{2}} \frac{\partial T}{\partial \theta}-2 \frac{\partial T}{\partial t}\right) A
\end{aligned}
$$




$$
+\frac{B^{2}}{r^{4}} \frac{\partial^{2} R}{\partial \theta^{2}}+\frac{B B^{\prime}}{r^{4}} \frac{\partial R}{\partial \theta}+\frac{2 B}{r^{2}} \frac{\partial^{2} R}{\partial \theta \partial t}+\frac{\partial^{2} R}{\partial t^{2}} .
$$

In equations (13) and (16) and in the sequel, a prime denotes total derivative with respect to $\theta$, so that $B^{\prime}=d B / d \theta$. The solutions of the equations (11]16) determine the Lie point symmetries of the reduced Ermakov system as well as the classes of admissible functions $A$ and $B$. In the following Section we show four categories of solutions for the determining equations.

\section{Exact solutions}

A closer examination of (13) shows that the function $A$ which specifies the dynamics of the radial variable in the reduced Ermakov system, will soon become, to some extent, determined if $\partial T / \partial r \neq 0$. Indeed, (11) and (12) immediately give the $r$ dependence of $R$ and $T$, which, in turn, will determine the $r$ dependence of $A$ through (13). Furthermore, using (14,15) shows that, for $\partial T / \partial r \neq 0, A$ contains only two terms, one proportional to $r$, the other to $r^{-3}$. To avoid this excessively constrained situation we must have

$$
\frac{\partial T}{\partial r}=0
$$

a condition assumed throughout this paper.

If (17) is valid, the solution for (11,15) is

$$
\begin{aligned}
R & =(\rho(t) \dot{\rho}(t)+\Gamma(\theta)) r \\
S & =S(\theta)=\left(\kappa-2 \int^{\theta} d \phi \frac{\Gamma(\phi)}{B(\phi)}\right) B(\phi), \\
T & =\rho^{2}(t),
\end{aligned}
$$

where $\rho$ is an arbitrary function of time, $\Gamma$ is an arbitrary function of $\theta$ and $\kappa$ a numerical constant.

Until now, no constraint was imposed on the functions $A$ or $B$ of the reduced Ermakov system, but there still remains the symmetry condition (16). Inserting (18) 20) into (16), we get the following determining equation,

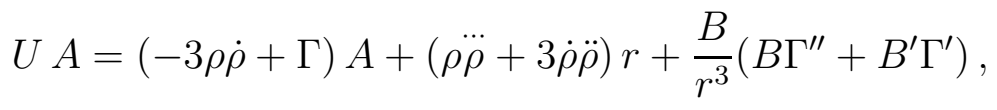


where $U$ is the generator of Lie point symmetries for reduced Ermakov systems,

$$
U=(\rho \dot{\rho}+\Gamma) r \frac{\partial}{\partial r}+S \frac{\partial}{\partial \theta}+\rho^{2} \frac{\partial}{\partial t} .
$$

The generator $U$, contains two arbitrary functions, $\rho(t)$ and $\Gamma(\theta)$, and one arbitrary numerical constant, $\kappa$, implicit in the definition (19) of $S(\theta)$.

The $\operatorname{sl}(2, R)$ algebra is obtained from (22) in the particular case

$$
\rho^{2}=c_{0}+c_{1} t+c_{2} t^{2}, \quad \Gamma=\kappa=0,
$$

where $c_{0}, c_{1}$ and $c_{2}$ are arbitrary numerical constants. The three generators of the $\operatorname{sl}(2, R)$ algebra is obtained by taking separately each of these constants non zero. In comparison with the generator of point symmetries of the non reduced Ermakov system [15, the new ingredients of $U$ are $\Gamma(\theta)$ and $\kappa$. Also, $\rho^{2}(t)$ is not necessarily a second degree polynomial in $t$. In Section 4 , we present a more detailed account of the relation between the point symmetries of the reduced Ermakov systems and the $s l(2, R)$ algebra.

Equation (21) can be viewed either as an equation for $A$ or for $B$. We feel it is more productive to think of it as a determining equation for $A$, since $B$ participates in the generator through the definition (19). Following this choice, we find four classes of solutions for $A$ satisfying (21), listed bellow. All these solutions are build using the differential invariants of the operator $U$, that is, the independent functions $I_{1}$ and $I_{2}$ for which $U\left(I_{1}\right)=U\left(I_{2}\right)=0$.

\subsection{The $\rho \neq 0, S \neq 0$ Case}

In this situation, the method of characteristics yields the following differential invariants for the generator $U$,

$$
\begin{aligned}
& I_{1}=\int^{\theta} \frac{d \phi}{S(\phi)}-\int^{t} \frac{d \tau}{\rho^{2}(\tau)}, \\
& I_{2}=\frac{r}{\rho} \exp \left(-\int^{t} \frac{d \tau}{\rho^{2}(\tau)} \Gamma\left(\theta\left(\tau ; I_{1}\right)\right)\right) .
\end{aligned}
$$

In (25),$\theta=\theta\left(t ; I_{1}\right)$ is a function of $t$ as given locally by the implicit function theorem through (24). The differential invariants can be used to construct the solution for (21). The result is

$$
A=\frac{\ddot{\rho}}{\rho} r+\frac{1}{\rho^{3}} \exp \left(\int^{t} \frac{\Gamma(\tau)}{\rho^{2}(\tau)} d \tau\right) \tilde{A}\left(I_{1}, I_{2}\right)+\frac{1}{r^{3}} \exp \left(4 \int^{t} \frac{\Gamma(\tau)}{\rho^{2}(\tau)} d \tau\right) \times
$$




$$
\times \int^{t} \frac{d \mu}{\rho^{2}(\mu)}\left(B^{2} \Gamma^{\prime \prime}+B B^{\prime} \Gamma^{\prime}\right)(\mu) \exp \left(-4 \int^{\mu} \frac{\Gamma(\nu)}{\rho^{2}(\nu)} d \nu\right),
$$

where $\theta$, in the integrals, is taken as a function of $t$ through (24) and the implicit function theorem. $\tilde{A}$ is an arbitrary function of the differential invariants $I_{1}$ and $I_{2}$.

To summarize, for any function $B$, the reduced Ermakov system (4] 5) has a Lie point symmetry with generator (22) with $\rho \neq 0$ and $S \neq 0$ provided $A$ can be cast in the form (26). While $B$ remains completely arbitrary, $A$ belongs to a large class of functions, including the arbitrary functions $\rho, \Gamma$ and $\tilde{A}$ and the numerical constant $\kappa$. Notice that the Ermakov invariant enters as a parameter in the symmetry generator as well as in the function $A$. This is no surprise since the Ermakov invariant was used to eliminate $\dot{\theta}$ from the equations of motion.

\subsection{The $\rho \neq 0, S=0$ Case}

Now the differential invariants for $U$ are

$$
I_{1}=\frac{r}{\rho}, \quad I_{2}=\theta
$$

and the corresponding solution for (21) is

$$
A=\frac{\ddot{\rho}}{\rho} r+\frac{1}{r^{3}} \tilde{A}\left(\frac{r}{\rho}, \theta\right)
$$

where $\tilde{A}$ is an arbitrary function of the indicated arguments. This class of solutions contains the arbitrary functions $\rho$ and $\tilde{A}$, subject to $\rho \neq 0$. Also, $B$ remains completely free and does not appear in the generator.

\subsection{The $\rho=0, \Gamma \neq 0$ Case}

The differential invariants are

$$
I_{1}=\left(\kappa-2 \int^{\theta} d \phi \frac{\Gamma(\phi)}{B(\phi)}\right)^{1 / 2} r, \quad I_{2}=t,
$$

while

$$
\begin{aligned}
A & =\frac{\kappa B(\theta)}{r^{3}}\left(\kappa-2 \int^{\theta} d \phi \frac{\Gamma(\phi)}{B(\phi)}\right)^{-2} \Gamma^{\prime}(\theta)+r \tilde{A}\left(I_{1}, I_{2}\right) \\
& -\frac{2}{r^{3}}\left(\kappa-2 \int^{\theta} d \phi \frac{\Gamma(\phi)}{B(\phi)}\right)^{-2} \int^{\theta} d \mu\left(B \Gamma^{\prime \prime}+B^{\prime} \Gamma^{\prime}\right)(\mu) \int^{\mu} d \nu \frac{\Gamma(\nu)}{B(\nu)}
\end{aligned}
$$


for $\tilde{A}$ an arbitrary function depending on the differential invariants of the symmetry generators. Now there are the free functions $\Gamma, B$ (which appears in the symmetry generator) and $\tilde{A}$, besides the numerical constant $\kappa$.

\subsection{The $\rho=\Gamma=0, \kappa \neq 0$ Case}

This is the most simple situation. The differential invariants are $r$ and $t$ and the solution for (21) is $A=A(r, t)$. The symmetry simply reflects the fact that $\theta$-independent equations are invariant under rotations.

\section{Connection with the $S L(2, R)$ Group}

$S L(2, R)$ is the Lie point symmetry group of non reduced Ermakov systems [15]-19], and we have to investigate the role of this transformations group for the reduced Ermakov systems. For simplicity, in this Section we consider Ermakov systems containing frequencies depending only on time. In this case (see equation (6) $) A$ is given by

$$
A(r, \theta, t)=-\Omega^{2}(t) r+\frac{1}{r^{3}}\left(F(\theta)+B^{2}(\theta)\right),
$$

for a time-dependent frequency $\Omega(t)$. Inserting (31) in the symmetry condition (21), we get

$$
\begin{aligned}
(\rho \dddot{\rho} & \left.+3 \dot{\rho} \ddot{\rho}+4 \Omega^{2} \rho \dot{\rho}+2 \Omega \dot{\Omega} \rho^{2}\right) r++\left(B^{2} \Gamma^{\prime \prime}+B B^{\prime} \Gamma^{\prime}+\right. \\
& \left.+4\left(F+B^{2}\right) \Gamma-\left(F^{\prime}+2 B B^{\prime}\right) S\right) r^{-3}=0,
\end{aligned}
$$

for $S$ defined in (19). Because (32) has to be satisfied for arbitrary $r$, it can be split in two parts, one corresponding to $r$, the other to $r^{-3}$,

$$
\begin{aligned}
& \ddot{\rho}+3 \ddot{\rho} \ddot{\rho}+4 \Omega^{2} \rho \dot{\rho}+2 \Omega \dot{\Omega} \rho^{2}=0, \\
& B^{2} \Gamma^{\prime \prime}+B B^{\prime} \Gamma^{\prime}+4\left(F+B^{2}\right) \Gamma=\left(F^{\prime}+2 B B^{\prime}\right) S \text {. }
\end{aligned}
$$

Equation (34) may be cast into a simpler form using a new independent variable $\varphi$ given by

$$
\varphi=\int^{\theta} \frac{d \mu}{B(\mu)}
$$


and a new dependent variable $W$ defined by

$$
\Gamma=\frac{d W}{d \varphi}
$$

Using the definition (19) of $S$ as well as introducing

$$
H=F+B^{2},
$$

equation (34) is converted into

$$
\frac{d^{3} W}{d \varphi^{3}}+4 H \frac{d W}{d \varphi}+2 \frac{d H}{d \varphi} W=\kappa \frac{d H}{d \varphi} .
$$

Equations (33) and (38) can be used to search for symmetries of specific reduced Ermakov systems. However, for arbitrary reduced Ermakov systems, that is, for completely arbitrary functions $H$ in (38), the only possibility is

$$
W=\kappa=0 .
$$

This will be the choice if we are interested in symmetries valid for all non reduced Ermakov systems, regardless this specific form. Notice that $W=0$ implies $\Gamma=0$ in the symmetry generator.

The other condition which remains is (33), which can be integrated once yielding Pinney's [23] equation,

$$
\ddot{\rho}+\Omega^{2} \rho=\frac{c}{\rho^{3}}
$$

where $c$ is a constant. However, this is a nonlinear equation, and a more fruitful approach for the study of symmetries is the linearizing transform

$$
a=\rho^{2},
$$

giving

$$
\dddot{a}+4 \Omega^{2} \dot{a}+4 \Omega \dot{\Omega} a=0 .
$$

According to (22), the solution for this equation determines the symmetry generator

$$
U=a \frac{\partial}{\partial t}+\frac{\dot{a} r}{2} \frac{\partial}{\partial r}
$$


Now, with the rescaling

$$
\alpha=a / \psi, \quad \tau=\int^{t} d \mu / \psi^{2}(\mu),
$$

where $\psi$ is any particular solution for the time-dependent harmonic oscillator equation

$$
\ddot{\psi}+\Omega^{2} \psi=0
$$

we transform (42) into

$$
\frac{d^{3} \alpha}{d \tau^{3}}=0
$$

The general solution is (compare with (23) $)$

$$
\alpha=c_{0}+c_{1} \tau+c_{2} \tau^{2}
$$

for constants $c_{0}, c_{1}$ and $c_{2}$. Taking separately each of these constants non zero, we obtain three symmetry generators for arbitrary reduced Ermakov systems. In the original, non rescaled variables and using (43), the corresponding symmetry generators are

$$
\begin{aligned}
U_{0} & =\psi^{2} \frac{\partial}{\partial t}+\psi \dot{\psi} r \frac{\partial}{\partial r} \\
U_{1} & =\psi^{2} \int^{t} \frac{d \mu}{\psi^{2}(\mu)} \frac{\partial}{\partial t}+\left(\frac{1}{2}+\psi \dot{\psi} \int^{t} \frac{d \mu}{\psi^{2}(\mu)}\right) r \frac{\partial}{\partial r} \\
U_{2} & =\psi^{2}\left(\int^{t} \frac{d \mu}{\psi^{2}(\mu)}\right)^{2} \frac{\partial}{\partial t}+ \\
& +\left(1+\psi \dot{\psi} \int^{t} \frac{d \mu}{\psi^{2}(\mu)}\right) \int^{t} \frac{d \mu}{\psi^{2}(\mu)} r \frac{\partial}{\partial r}
\end{aligned}
$$

Calculating the Lie brackets, the result is

$$
\left[U_{0}, U_{1}\right]=U_{0}, \quad\left[U_{0}, U_{2}\right]=2 U_{1}, \quad\left[U_{1}, U_{2}\right]=U_{2},
$$

which is the $\operatorname{sl}(2, R)$ algebra. This shows that the symmetry group for arbitrary reduced Ermakov systems is $S L(2, R)$. It is interesting to note that the algebra of the vector fields $U_{0}, U_{1}$ and $U_{3}$ is $\operatorname{sl}(2, R)$ regardless the form of $\psi$ (does not need to be a solution of a time-dependent harmonic oscillator). In addition, we notice that $U_{0}, U_{1}$ and $U_{2}$ do not depend on the Ermakov invariant, being generators of point transformations also in the non reduced space. 


\section{An Illustrative Example}

Let us consider a non reduced Ermakov system with

$$
G(\theta)=-\frac{L^{2} \sin \theta}{\cos ^{3} \theta}, \quad L=\text { constant }
$$

in equation (2), producing an Ermakov invariant

$$
I=\frac{1}{2}\left(r^{2} \dot{\theta}\right)^{2}+\frac{L^{2}}{2 \cos ^{2} \theta}
$$

and a reduced Ermakov system with

$$
B(\theta)=\frac{\sqrt{2 I}}{\cos \theta}\left(1-\frac{L^{2}}{2 I}-\sin ^{2} \theta\right)^{1 / 2} .
$$

We look for functions $A(r, \theta, t)$ in the reduced Ermakov system that lead to Lie point symmetries as described by the class of solutions in subsection 3.1. Other classes of solutions may also be studied, but we restrict ourselves to this case in view of its generality. In fact, the solutions described in 3.1 have too many arbitrary functions, and we restrict ourselves to the choices

$$
\kappa=0, \quad \Gamma(\theta)=\Gamma_{0} \sin \theta, \quad \rho(t)=\cos \omega_{0} t,
$$

for constants $\Gamma_{0}$ and $\omega_{0}$.

In order to explicitly write the solution $A$, we have first to obtain the differential invariants (24]25) of the Lie symmetry. According to (19) and (54,55), we have

$$
S(\theta)=\frac{2 \Gamma_{0}}{\cos \theta}\left(1-\frac{L^{2}}{2 I}-\sin ^{2} \theta\right),
$$

which, substituted in (24), gives the differential invariant

$$
I_{1}=\frac{1}{2 \Gamma_{0}} \frac{1}{\sqrt{1-L^{2} / 2 I}} \operatorname{arctanh}\left(\frac{\sin \theta}{\sqrt{1-L^{2} / 2 I}}\right)-\frac{\tan \omega_{0} t}{\omega_{0}} .
$$

To obtain the second differential invariant from (25), we have to solve (157) for $\theta$, which in this case yields

$$
\theta=\arcsin \left(\sqrt{1-\frac{L^{2}}{2 I}} \tanh \left(2 \Gamma_{0} \sqrt{1-\frac{L^{2}}{2 I}}\left(\frac{\tan \omega_{0} t}{\omega_{0}}+I_{1}\right)\right)\right)
$$


With this result and performing the necessary quadratures, we find from (25) the differential invariant

$$
I_{2}=\left(1-\frac{\sin ^{2} \theta}{1-L^{2} / 2 I}\right)^{1 / 4} \frac{r}{\cos \omega_{0} t} .
$$

Again using (58) and performing the necessary integrations in (26), we find the set of admissible $A$ functions given by

$$
A=-\omega_{0}^{2} r-\frac{\left(1-L^{2} / 2 I\right) \sin ^{2} \theta}{\left(1-L^{2} / 2 I-\sin ^{2} \theta\right)} \frac{I}{r^{3}}+\frac{r}{\cos ^{4} \omega_{0} t} \tilde{A}\left(I_{1}, I_{2}\right)
$$

for arbitrary $\tilde{A}$ depending on the differential invariants at (57) and (59). We have redefined the arbitrary function $\tilde{A}$ in (26) by making $\tilde{A} \rightarrow \tilde{A} / I_{2}$ for the sake of a better notation.

To summarize, reduced Ermakov systems (44-5) with $A$ given by (60) and $B$ given by (54) do possess Lie point symmetries with generator

$$
\begin{aligned}
U & =\left(-\omega_{0} \sin \omega_{0} t \cos \omega_{0} t+\Gamma_{0} \sin \theta\right) r \frac{\partial}{\partial r}+ \\
& +\frac{2 \Gamma_{0}}{\cos \theta}\left(1-\frac{L^{2}}{2 I}-\sin ^{2} \theta\right) \frac{\partial}{\partial \theta}+\cos ^{2} \omega_{0} t \frac{\partial}{\partial t},
\end{aligned}
$$

as follows from (22) and (55) (56). A remark applicable here and in most of other cases is that the Ermakov invariant does appear in the generator of symmetries. This is no surprise since the reduced Ermakov system was written after restricting the trajectories to the level surfaces of the Ermakov invariant. Therefore, the reduced Ermakov systems contains $I$ as a parameter. Hence, (61) is the generator of a point transformation on the reduced space and of a dynamical transformation on the non reduced space, where the Ermakov invariant is written as a function of $r, \theta$ and $\dot{\theta}$ as in (53).

\section{Application to the Generalized Paul Trap}

In practical applications, more often one does not assume the form of the generator and try to obtain the equations of motion for which it is a symmetry, like in Section 5. Instead, one has some specific equations of motion and then look for symmetries. This is the approach we follow in this Section, searching for Lie point symmetries for the following class of Ermakov 
systems, written in cartesian coordinates:

$$
\begin{aligned}
& \ddot{x}+\left(\Omega^{2}(t)-\frac{\varsigma(x \dot{y}-y \dot{x}, y / x)}{\left.\left(x^{2}+y^{2}\right)^{3 / 2}\right) x}=\frac{L^{2}}{x^{3}},\right. \\
& \ddot{y}+\left(\Omega^{2}(t)-\frac{\varsigma(x \dot{y}-y \dot{x}, y / x)}{\left(x^{2}+y^{2}\right)^{3 / 2}}\right) y=0,
\end{aligned}
$$

where $\Omega$ and $\varsigma$ are initially arbitrary functions of the indicated arguments, and $L$ is a constant. For constant $\Omega$ and $\varsigma$, these are the equations of motion for an ion in the presence of a Paul trap [24] with equal secular frequencies. In this context, we call (62,63) the equations of motion for a generalized Paul trap. Paul traps are a standard configuration used in ion trapping experiments [25]. Also notice that, for $\varsigma$ depending only on $y / x$, equations (62) 63) is a particular case of the Kepler-Ermakov systems, which are linearizable through point transformations [13, 22]. Here we ask for the classes of functions $\Omega$ and $\varsigma$ for which the corresponding reduced Ermakov systems do admit Lie point symmetries.

In polar coordinates, the generalized Paul trap equations can be cast in the Ermakov form (10) with

$$
F(\theta)=\frac{L^{2}}{\cos ^{2} \theta}, \quad G(\theta)=-\frac{L^{2} \sin \theta}{\cos ^{3} \theta}, \quad \omega^{2}=\Omega^{2}(t)-\frac{\varsigma\left(r^{2} \dot{\theta}, \tan \theta\right)}{r^{3}} .
$$

Notice the generalized character of $\omega$, which is not necessarily a function of time only.

The associated Ermakov invariant is

$$
I=\frac{1}{2}\left(r^{2} \dot{\theta}\right)^{2}+\frac{L^{2}}{2 \cos ^{2} \theta}
$$

and the reduced Ermakov system is constructed with the functions

$$
\begin{aligned}
A(r, \theta, t) & =-\Omega^{2}(t) r+\frac{\sigma(\theta)}{r^{2}}+\frac{2 I}{r^{3}}, \\
B(\theta) & =\frac{\sqrt{2 I}}{\cos \theta}\left(1-\frac{L^{2}}{2 I}-\sin ^{2} \theta\right)^{1 / 2},
\end{aligned}
$$

where we have defined

$$
\sigma(\theta)=\varsigma(B(\theta), \tan \theta) .
$$

Let us search for symmetries of the type shown in Section 3. For this, a convenient approach is to substitute $A$ and $B$ in the symmetry condition 
(21) looking for some symmetry generator. The symmetry condition (21) then gives

$$
\begin{aligned}
(\dddot{\rho}+ & \left.+3 \dot{\rho} \ddot{\rho}+4 \Omega^{2} \rho \dot{\rho}+2 \Omega \dot{\Omega} \rho^{2}\right) r+\left(-S(\theta) \sigma^{\prime}+(3 \Gamma(\theta)-\rho \dot{\rho}) \sigma\right) r^{-2} \\
& +\left(\frac{2 I\left(1-L^{2} / 2 I-\sin ^{2} \theta\right)}{\cos ^{2} \theta} \Gamma^{\prime \prime}-\frac{L^{2} \sin \theta}{\cos ^{3} \theta} \Gamma^{\prime}+8 I \Gamma\right) r^{-3}=0,
\end{aligned}
$$

where $S(\theta)$ depends on $\Gamma(\theta)$ according to (19). Compare (69) with (32). There are similarities since $B(\theta)$ here is the same as in Section 5 , but now there is a contribution proportional to $r^{-2}$, peculiar to Kepler-Ermakov systems.

As in Section 5, we split the symmetry condition (69) into three equations, corresponding to different powers of $r$,

$$
\begin{aligned}
\ddot{\rho}+3 \dot{\rho} \ddot{\rho}+4 \Omega^{2} \rho \dot{\rho}+2 \Omega \dot{\Omega} \rho^{2} & =0, \\
S(\theta) \sigma^{\prime}+(\rho \dot{\rho}-3 \Gamma(\theta)) \sigma & =0, \\
\frac{2 I\left(1-L^{2} / 2 I-\sin ^{2} \theta\right)}{\cos ^{2} \theta} \Gamma^{\prime \prime} & -\frac{L^{2} \sin \theta}{\cos ^{3} \theta} \Gamma^{\prime}+8 I \Gamma=0 .
\end{aligned}
$$

For consistency with equation (68), in equation (71) we must have

$$
\rho \dot{\rho}=\frac{\Omega_{0}}{2},
$$

for a constant $\Omega_{0}$. Integrating, we get

$$
\rho=\left(\rho_{0}^{2}+2 \Omega_{0} t\right)^{1 / 2},
$$

where $\rho_{0}$ is a constant. Inserting this into (70), we obtain the following class of frequencies $\Omega$,

$$
\Omega=\frac{\Omega_{0}}{\rho_{0}^{2}+2 \Omega_{0} t} .
$$

Equation (72) can be best handled using the new independent variable

$$
\varphi=\int^{\theta} \frac{d \mu}{B(\mu)}=\frac{1}{\sqrt{2 I}} \arcsin \left(\frac{\sin \theta}{\sqrt{1-L^{2} / 2 I}}\right),
$$

yielding

$$
\frac{d^{2} \Gamma}{d \varphi^{2}}+8 I \Gamma=0,
$$


with solution

$$
\Gamma=\Gamma_{1} \cos (2 \sqrt{2 I} \varphi)+\Gamma_{2} \sin (2 \sqrt{2 I} \varphi),
$$

where $\Gamma_{1}$ and $\Gamma_{2}$ are constants.

There remains the equation (71). Calculating $S(\theta)$ via (19) and using the new independent variable $\varphi$, this equation reads

$$
\left(\kappa+\frac{\Gamma^{\prime}}{4 I}\right) \frac{d \sigma}{d \varphi}+\left(\Omega_{0}-3 \Gamma\right) \sigma=0
$$

with $\Gamma$ as in (78).

There is no difficulty at solving (179) in the general case, but the solution is somewhat complicated. It seems more illustrative to obtain the general solution in some specific cases, listed bellow.

\subsection{The $\kappa \neq 0, \Gamma_{1}=\Gamma_{2}=0$ Case}

The general solution for (779) is

$$
\sigma=\sigma_{0} e^{-\frac{\Omega_{0} \varphi}{\kappa}}=\sigma_{0} \exp \left(-\frac{\Omega_{0}}{\kappa \sqrt{2 I}} \arcsin \left(\frac{\sin \theta}{\sqrt{1-L^{2} / 2 I}}\right)\right),
$$

for any constant $\sigma_{0}$.

\subsection{The $\Gamma_{1}^{2}+\Gamma_{2}^{2} \neq 0, \kappa=\Omega_{0}=0$ Case}

In this situation the general solution is

$$
\sigma=\sigma_{0}\left(\frac{d \Gamma}{d \varphi}\right)^{-3 / 2}
$$

where $\sigma_{0}$ is a constant and $\Gamma$ is given by (78), with $\varphi$ defined in (76)).

In both subcases (80) and (81), the solution contains the Ermakov invariant (65), which is dependent on $r^{2} \dot{\theta}$. Hence, at least in the generalized Paul trap case, we have not found solutions for which $\sigma$ is a function of $\theta$ only, characterizing a Kepler-Ermakov system.

To summarize, we found the functions $\sigma$ (alternatively, $\varsigma$ ) and $\Omega$ so that the reduced Ermakov system for the generalized Paul trap equations do possess Lie point symmetries. The functions $\sigma$ do satisfy (79), as in subsections 6.1 and 6.2 , while the frequency $\Omega$ is of the form (75). The corresponding generator of symmetry is build with $\rho$ given by (74) and $\Gamma$ given by (78). 


\section{Conclusion}

We presented the general treatment for Lie point symmetries of reduced Ermakov systems. We found four classes of reduced Ermakov systems possessing Lie point symmetries, all of them involving arbitrary functions. We have applied the results to two different systems, including a generalized Paul trap. From the theoretical viewpoint, the most important result we have found is the fact that the $S L(2, R)$ group is more exactly a property of the reduced Ermakov system. For Lagrangian Ermakov systems, the existence of the Ermakov invariant follows from a dynamical symmetry. Then, for reduced Lagrangian Ermakov systems, the symmetry structure can be separated in two distinct parts: a dynamical symmetry leading to the Ermakov invariant, and the $S L(2, R)$ group for the reduced Ermakov system, the later a consequence of the first symmetry.

\section{References}

[1] Ermakov V. P. 1880 Univ. Izv. Kiev 201.

[2] Ray J. R. and Reid J. L. 1979 Phys. Lett. A 71317.

[3] Lewis H. R. 1967 Phys. Rev. Lett. 18510.

[4] Haas F. 2002 Phys. Rev. A 65033603.

[5] Lidsey J. E. 2004 Class. and Quantum Gravity 21777.

[6] Hawkins R. M. and Lidsey J. E. 2002 Phys. Rev. D 66023523.

[7] Ioffe M. V. and Korsch H. J. 2003 Phys. Lett. A 311200.

[8] Rosu H. C. 2002 Physica Scripta 65296.

[9] Akulov V. P, Catto S., Cebioğlu O. and Pashnev A. 2003 Phys. Lett. B 575137.

[10] Haas F. 2002 J. Phys. A: Math. Gen. 352925.

[11] Reid J. L. and Ray J. R. 1980 J. Math. Phys. 211583. 
[12] Athorne C., Rogers C., Ramgulam U. and Osbaldestin A. 1990 Phys. Lett. A 143207.

[13] Haas F. and Goedert J. 1999 J. Phys. A: Math. Gen. 322835.

[14] Olver P. J. 1986 Applications of Lie Groups to Differential Equations (Springer-Verlag: Berlin).

[15] Leach P. G. L. 1991 Phys. Lett. A 158102.

[16] Govinder K. S., Athorne C. and Leach P. G. L. 1993 J. Phys. A: Math. Gen. 264035.

[17] Govinder K. S. and Leach P. G. L. 1994 J. Phys. A: Math. Gen. 27 4153.

[18] Govinder K. S. and Leach P. G. L. 1994 Phys. Lett. A 186391.

[19] Goedert J. and Haas F. 1998 Phys. Lett. A 239348.

[20] Haas F. and Goedert J. 2001 Phys. Lett. A 279181.

[21] Karasu A. and Yildirim H. 2002 J. Nonlinear Math. Phys. 9475.

[22] Athorne C. 1991 J. Phys. A: Math. Gen. 24 L1385.

[23] Pinney E. 1950 Proc. Am. Math. Soc. 1681.

[24] Paul W. and Steinwedel H. 1953 Z. Naturforschg. 8a 448.

[25] Baumman G. and Noemacher T. F. 1992 Phys. Rev. A 462682. 